\title{
Achieving High-Bandwidth Nanopositioning In Presence of Plant Uncertainties
}

\author{
Sumeet S. Aphale \\ ARC Centre for Complex \\ Dynamic Systems and Control \\ University of Newcastle \\ Callaghan, Australia, NSW 2308 \\ Email: sumeet.aphale@newcastle.edu.au
}

\author{
Santosh Devasia \\ Mechanical Engineering Department \\ University of Washington \\ Seattle, WA, USA \\ Email: sdevasia@u.washington.edu
}

\author{
S. O. Reza Moheimani \\ School of Electrical and \\ Computer Engineering \\ University of Newcastle \\ Callaghan, Australia, NSW 2308 \\ Email: reza.moheimani@newcastle.edu.au
}

\begin{abstract}
In the absence of plant parameter uncertainties, inversion-based feedforward techniques have been known to deliver accurate tracking performance. Due to changes in operating conditions like ambient temperature, humidity and loading, piezoelectric-stack actuated nanopositioning platforms can undergo significant changes in their system parameters. Nonlinear effects of hysteresis, an inherent property of a piezoelectric actuator, are also present; charge actuation is applied to reduce the effects of hysteresis. In this work, a suitable feedback controller that reduces the effects of parameter uncertainties is integrated with the inversion-based feedforward technique to deliver accurate nanopositioning over a large bandwidth. It is shown experimentally that by integrating closed-loop damping, inversion-based feedforward and charge actuation, the tracking bandwidth of the platform from can be increased significantly from $310 \mathrm{~Hz}$ to 1320 Hz.
\end{abstract}

Index Terms-Nanopositioning, Feedforward, Feedback, highspeed tracking

\section{INTRODUCTION}

Due to their simple and robust construction, low crosscoupling between the axes and substantially large motion ranges, piezoelectric-stack actuated nanopositioning platforms are gaining popularity in many nanopositioning applications. They have a relatively narrow tracking bandwidth due to the highly resonant low-frequency mode (in the hundreds of Hertz) dominating their frequency response. As a result, significant research interest has been genereted by control strategies that can increase the tracking precision and bandwidth of these platforms.

Various nano-scale positioning approaches have been researched in the past. Salapaka et. al. reported robust tracking in [1]. Force-feedback for nanomanipulation was investigated by Sitti et. al. in [2]. Sebastian et. al. researched $H_{\infty}$ control algorithms for nano-scale tracking [3]. Inversion-based feedforward techniques for continuously tracking a desired trajectory have been under investigation [4], [5]. Zhou et. al. applied inversion-based feedforward technique to nanopositioning systems [6]. Compensating for the nonlinear effects of hysteresis and creep using inversion-based feedforward was reported in [7], [8]. High sensitivity to modeling errors and plant parameter uncertainties are the two main drawbacks of the feedforward control strategy. Feedback has been shown to improve the bandwidth of feedforward strategies by suppressing the effects of uncertainties [9]. An in-depth overview of this field has been documented in [10], [11].
Change in resonance frequency is a commonly occurring system uncertainty for the positioning stage. Change in ambient temperature, humidity, atmospheric pressure and mechanical loading are some of the reasons that contribute to changes in resonance frequencies. The most effective method to make the system less sensitive to this change in resonance frequencies is to damp the structure using a feedback controller. Passive damping techniques such as shunts have been effective but may need constant tuning to avoid performance degradation under resonance frequency changes, thus defeating the initial purpose of using one. To handle these system uncertainties, more evolved adaptive shunts are needed [12], [13]. Various feedback controllers that impart substantial damping to the system have been formulated and documented over the years [14], [15], [16]. Positive Position Feedback control (PPF) [17] and Resonant control [18] have all shown substantial damping capability. A polynomial-based controller is one of the simplest control design method that imparts substantial damping to the system [19]. This controller is easy to construct for second-order plants and is robust under resonance frequency variations as documented in [20], [21]. Combining such damping controllers that are insensitive to change in resonance frequencies with a Proportional Integral controller (PI) has been reported earlier [22]. The high gain needed by the integrator to track a given trajectory limits the achievable scan speed using this technique to $4 \mathrm{~Hz}$.

In this paper, the polynomial-based damping controller is integrated with the inversion-based feedforward technique. The polynomial-based damping controller increases plant insensitivity to changes in resonance frequency and thus, when combined with the inversion-based feedforward technique achieves a much larger positioning bandwidth with substantial tracking accuracy. A custom-built charge amplifier capable of driving the high capacitive loads presented by the piezoelectric stacks $(\approx$ $10 \mu \mathrm{f}$ ) is employed to reduce the nonlinear effects of hysteresis present in piezoelectric stacks. An $89 \%$ reduction in hysteresis has been reported due to the charge actuation approach in [23].

\section{A. Objectives and Outline}

The main objective of this work is to increase the positioning bandwidth of a typical piezoelectric-stack actuated nanopositioning platform using closed-loop damping and inversionbased feedforward techniques. Section II provides details of 


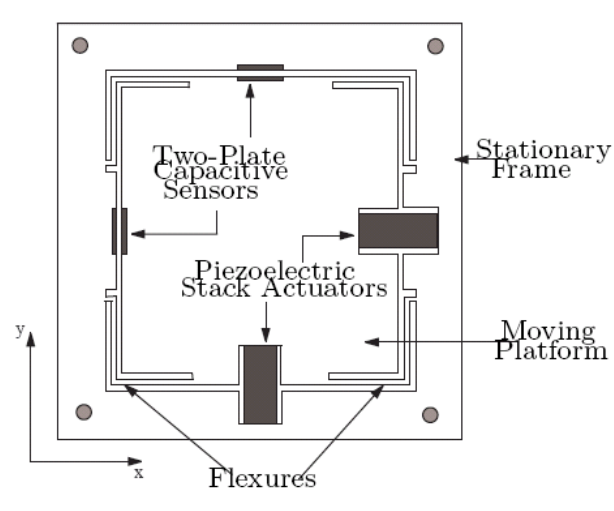

(a)

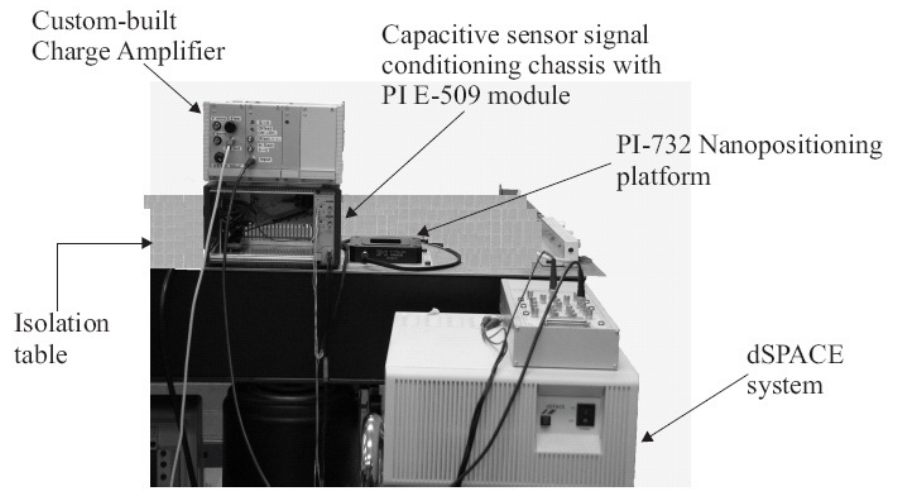

(b)

Fig. 1. (a) Working principle of the monolithic XY piezoelectric-stack actuated nanopositioning platform.(b) A snapshot of the experimental setup used in this work

the experimental setup used in this work as well as the system identification carried out in lieu with this work. Section III will begin by discussing the inversion-based feedforward technique and its achievable positioning bandwidth. The drawbacks of using feedforward alone will be enumerated and possible solutions will be put forth. This will serve as the motivation for using a suitable feedback controller in conjunction with the feedforward technique. The improvement in positioning bandwidth due to this approach will be presented. Experimental results that validate the expected improvement in positioning bandwidth are presented in Section IV. Section V concludes the article.

\section{EXPERIMENTAl SETUP AND System IdENTIFICATION}

We use a two-axis piezoelectric stack-actuated platform based on a parallel-kinematic design, manufactured by PI (PI734). This design provides mounting independent orthogonality and reduced cross-coupling between the two axes. Friction and stiction are eliminated by using a flexure guidance system. The platform is equipped with a built-in integrated lever motion amplifier to increase the range of motion whilst maintaining the sub-nanometer accuracy. Each axis of the nanopositioning platform is fitted with a two-plate capacitive sensor that provides a direct position measurement. A simplified diagram of the nanopositioning platform is given in Figure 1 (a). The piezoelectric stack actuators take voltage input in the range of 0 $\mathrm{V}-100 \mathrm{~V}$ for each axis. The resultant motion produced by the platform is within $0 \mu \mathrm{m}-100 \mu \mathrm{m}$. This motion is detected by the two-plate capacitive sensors and fed to an electronic sensor output module. The output of this module is within $0 \mathrm{~V}-6.7$ $\mathrm{V}$.

A dSPACE-1005 rapid prototyping system equipped with 16bit ADC(DS2001) and DAC(DS2102) cards is used to implement the proposed strategy. The sampling frequency of this system is $20 \mathrm{kHz}$. An in-house, custom built charge amplifier with an equivalent voltage gain of 20 was used to drive the piezoelectric stack actuator. The charge amplifier is calibrated such that the voltage gain it provides remains constant in the voltage as well as charge mode. To measure the frequency response of one axis of the nanopositioning platform, a band- limited random noise input signal was generated by an HP signal analyzer, of amplitude $1 V_{p k}$ within the frequency range of $10 \mathrm{~Hz}-3.2 \mathrm{kHz}$. This signal was fed to the charge amplifier as input and the equivalent charge output of the amplifier was used to excite the piezoelectric stack. This input corresponds to a displacement within $0-1 \mu \mathrm{m}$, or $1 \%$ of the total range of the platform. Throughout this work, the piezoelectric stack actuator was kept at a bias of $+40 \mathrm{~V}$, to avoid depolarization due to application of negative voltages. As the identification was done from $10 \mathrm{~Hz}$ onwards, the effect of creep was eliminated due to the absence of any low-frequency components. The piezoelectric stack on the other axis is shorted out to eliminate its effect. The input is a charge signal applied to the piezoelectric stack ( $\mathrm{u}$ in $\mathrm{nC}$ ), and the output is the displacement ( $\mathrm{d}$ in $\mu \mathrm{m}$ ) obtained by scaling the measured capacitive sensor voltages by the proportional scaling factor $(0.067 \mathrm{~V} / \mu \mathrm{m})$. A picture of the complete setup is given in Figure 1(b).

A subspace-based modeling technique, [24], is used to obtain accurate models of the open- and closed-loop system. Fig 2 shows the measured frequency response for the open-loop system. A subspace-based second-order model accurately captures the system dynamics in the bandwidth of interest and is used throughout this work for further analysis and computations.

\section{FEEDFORWARD AND FEEDBACK}

This section briefly presents both the feedforward and the feedback algorithms. The polynomial-based controller designed to damp the platform resonant mode is also discussed in brief. For a more detailed explaination, the reader is referred to [25].

\section{A. Inversion-based feedforward technique}

The goal of the inversion-based feedforward technique is to find the input ' $u$ ' that when fed to a linear system with known dynamics ' $G_{x x}$ ' produces the desired output ' $y_{d}$ '. In other words, it is desirable to acquire a $u$ such that

$$
Y_{d}(\mathrm{i} \omega)=G_{x x}(\mathrm{i} \omega) U(\mathrm{i} \omega)
$$

where $U(\mathrm{i} \omega)$ and $Y_{d}(\mathrm{i} \omega)$ are Fourier transforms of $u$ and the desired trajectory $y_{d}$ respectively. 
Due to practical issues such as sensor and actuator bandwidth, ambient noise, sampling, quantization and machine precision the system can be accurately identified for only a finite bandwidth. As the system is identified over a finite bandwidth, it is logical to construct inputs which are also strictly bandlimited. Thus, the desired trajectory $y_{d}$ is shaped, using either a suitable filter or truncation of terms, such that it is bandlimited and all or most of its frequency content lies within the bandwidth of the identified system. In this work, we construct scan trajectories whose frequency components lie totally within the bandwidth of the identified system model, i.e. are strictly bandlimited.

If $G(s)$ is the actual system and $G_{m}(s)$ is the identified model then, due to practical limitations such as machine accuracy and limit on model order, there are bound to be errors in the identified model with respect to the actual system. Let this error be given by $\Delta(s)=G_{m}(s)-G(s)$. Then, it has been shown earlier that feedforward always improves output tracking for any feedback as long as $|\Delta(\omega)|<\left|G_{m}(\omega)\right|$, [26]. In the presence of such uncertainty, the achievable positioning bandwidth of the platform is substantially reduced. To elaborate this further, the resonance frequency of the platform is changed from $426 \mathrm{~Hz}$ to $350 \mathrm{~Hz}$ by manually loading it with some mass. Figure 2 shows the positioning bandwidth achievable by the inversionbased feedforward technique in the presence of uncertainty. As seen, the positioning bandwidth is limited to $310 \mathrm{~Hz}$. Feedback controllers aid in improving the tracking performance of the feedforward control techniques provided they reduce the sensitivity of the system to uncertainties such as changes in resonance frequency.

\section{B. Polynomial-based controller design}

For a system $G_{x x}$ whose transfer function is of the form

$$
G_{x x}==\frac{k_{x}}{s^{2}+2 \sigma_{x} \omega_{x} s+\omega_{x}^{2}}+D_{x},
$$

where $D_{x}$ is a feed-through term, a polynomial-based controller is defined by the second order transfer-function, [21],

$$
K_{\text {Poly }}(s) \triangleq \frac{\Gamma_{1} s+\Gamma_{2}}{s^{2}+2 \xi w s+w^{2}},
$$

where $\xi, w, \Gamma_{1}$ and $\Gamma_{2}$ are the design parameters. For the axis of interest, the polynomial-based controller design is described below. Note that poles of $G_{x x}(s)$, computed, are

$$
p_{ \pm}=-33.8 \pm \mathrm{i} 2644.6 \text {. }
$$

Here, the desired closed-loop poles are set to

$$
\begin{aligned}
& P_{1+}=P_{2+}=-2030.23 \pm \mathrm{i} 2644.5, \\
& P_{1-}=P_{2-}=-2030.23 \pm \mathrm{i} 2644.5,
\end{aligned}
$$

which amounts to placing the closed-loop poles of the system further into the left half plane by about 2000 units. It can be checked that the polynomial coefficients corresponding to the desired poles (5) are $K_{1}=8.1352 \times 10^{3}, K_{2}=3.8806 \times 10^{7}$, $K_{3}=9.0548 \times 10^{10}$ and $K_{4}=1.2388 \times 10^{14}$.
Solving for the controller parameters $\Gamma_{1}, \Gamma_{2}, \xi$ and $w$ gives the controller

$$
K_{\text {Poly }}(s) \triangleq \frac{-3678 s+1.0910^{6}}{s^{2}+7594 s+3.267 \times 10^{7}},
$$

that would render a closed-loop system having poles at $P_{1+}, P_{2+}, P_{1-}$ and $P_{2-}$. This controller damps the resonant mode of the system by $23 \mathrm{~dB}$, as shown.

Figures 2 and 3 plot the difference in frequency response of the unloaded and the loaded system for both the the undamped (open-loop) and the damped (closed-loop) configurations. It is clear that the unloaded and loaded system responses differ in open-loop undamped configuration by a substantial amount. On the other hand, there is minimal change in the equivalent closed-loop responses when the resonance has been damped using a polynomial-based controller. Applied to the open-loop (undamped) system, the inversion-based feedforward technique can only achieve a bandwidth of $310 \mathrm{~Hz}$. On the other hand, when feedback (closed-loop damped) is combined with the feedforward technique, the positioning bandwidth of the system increases substantially. It is seen that for the closed-loop plots shown in Figure 3(b), $\Delta_{c l}(\omega)<G_{c l}(\omega)$ for $\omega<1320 H z$. Thus, the positioning bandwidth has been increased by more than $1 \mathrm{kHz}$. A $5^{\text {th }}$-order model accurately captures the closedloop plant dynamics up to this frequency and is used for the inversion-based input generation. The overall control strategy implemented to achieve the high positioning bandwidth is shown in Figure 4.

\section{EXPERIMENTAL RESULTS}

A charge input equivalent to $15 V_{p k-p k}$ was used to ensure that the current limit of the charge amplifier is not reached during the experiments. This corresponds to a $15 \mu \mathrm{m}$ scan range. We have used the triangle waveforms as our reference for comparison as they are commonly used as desired trajectories for scanning applications,. It is known that the triangular waveform has infinite bandwidth and accurate tracking of this waveform is not practically possible. In general, a triangle wave can be approximated with additive synthesis, by adding odd harmonics of the fundamental, multiplying every $(4 n-1)^{t h}$ harmonic (where $n=1,2, \ldots$ ) by -1 (or changing its phase by $\pi$ ), and rolling off the harmonics by the inverse square of their relative frequency to the fundamental, see Eqns. (1 - 4). A quantification of how close this approximation is to the perfect triangle wave, when truncated at different harmonic components, is given in Table I. From the table, we can say that if the scan signal is constructed using the first 2 odd harmonics $\left(1^{s t}\right.$ and $\left.3^{r d}\right)$ of the fundamental then, $6.84 \%$ of the total scan will be within $1 \%$ of the perfect triangle. Similarly, if the scan signal is constructed using the first 7 odd harmonics $\left(1^{s t}, 3^{r d}\right.$, $5^{\text {th }}, 7^{\text {th }}, 9^{\text {th }}$ and $13^{\text {th }}$ ) then, $90.72 \%$ of the total scan will be within $1 \%$ of the perfect triangle. The rest of the table can be read in the same fashion.

It should be noted that perfect inversion will result in perfect tracking. As perfect inversion is impossible (due to plant uncertainty) and the system is identified for a limited bandwidth, the model suffers from unknown high-frequency dynamics. Due to the sensor noise, quantization noise from the ADC/DAC system and the modeling errors (the open-loop is a second-order model 

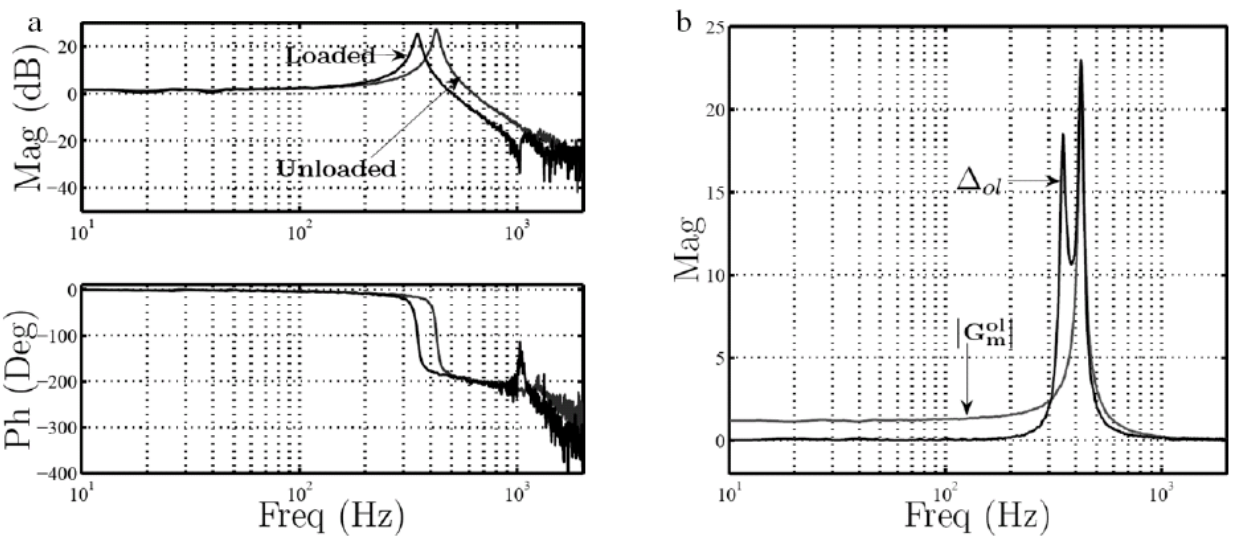

Fig. 2. (a) Open-loop frequency response for the unloaded and the loaded platform. Note that after loading, the resonance frequency shifts from $426 \mathrm{~Hz}$ to 350 $\mathrm{Hz}$ (b) A plot of modeling error due to plant uncertainty $\left|\Delta_{o l}\right|$ and the plant model used for inversion $\left|G_{m}(w)\right|$. As can be seen from the plot, $|\Delta(w)|>\left|G_{m}(w)\right|$ at $w=310 \mathrm{~Hz}$. Thus, the maximum positioning bandwidth achievable in the presence of system parameter uncertainty using feedforward alone is $310 \mathrm{~Hz}$.
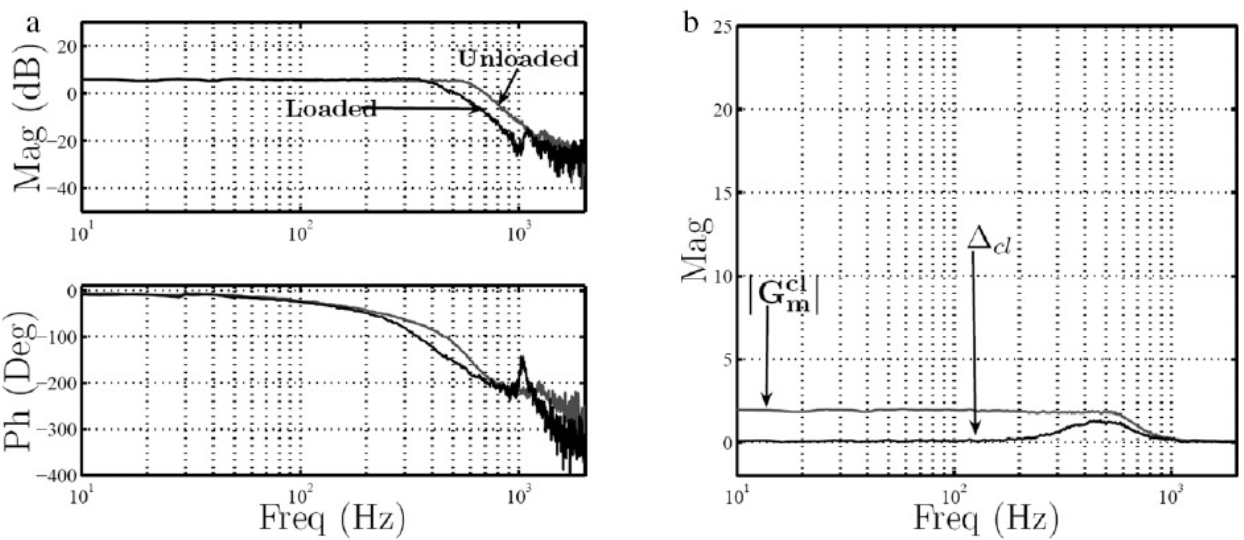

Fig. 3. (a) Closed-loop frequency response for the unloaded and the loaded platform. (b) A plot of modeling error due to plant uncertainty $\left|\Delta_{c l}\right|$ and the plant model used for inversion $\left|G_{m}(\omega)\right|$. As can be seen from the plot, $|\Delta(\omega)|>\left|G_{m}(\omega)\right|$ at $\omega=1320$ Hz. Thus, the maximum positioning bandwidth achievable in the presence of system parameter uncertainty using feedforward and feedback to damp the system resonance is $1320 \mathrm{~Hz}$. This is an increase in positioning bandwidth of more than $1 \mathrm{kHz}$ compared to the one achieved by feedforward alone.

\begin{tabular}{|c|c|c|c|}
\hline $\begin{array}{l}\text { Number of odd harmonics includ- } \\
\text { ing the fundamental }\end{array}$ & $\begin{array}{l}\text { Percentage of scan within 1\% er- } \\
\text { ror }\end{array}$ & $\begin{array}{l}\text { Percentage of scan within 2\% er- } \\
\text { ror }\end{array}$ & $\begin{array}{l}\text { Percentage of scan within 5\% er- } \\
\text { ror }\end{array}$ \\
\hline 2 & 6.84 & 15.92 & 94.40 \\
\hline 3 & 12.38 & 74.24 & 98.24 \\
\hline 4 & 77.44 & 96.48 & 99.96 \\
\hline 5 & 83.12 & 97.72 & 100 \\
\hline 6 & 85.12 & 98.52 & 100 \\
\hline 7 & 90.72 & 99.4 & 100 \\
\hline
\end{tabular}

TABLE I

TABLE SHOWING THE PERCENTAGE OF SCAN WITHIN A PARTICULAR ERROR RANGE FROM THE PERFECT TRIANGLE

and the closed-loop is a fifth-order model), the measured scans will have a higher level of error and therefore a lower acceptable scan range than that given in Table I. Scans with three different fundamental frequencies $(10 \mathrm{~Hz}, 40 \mathrm{~Hz}$ and $100 \mathrm{~Hz})$ each with two different number of harmonics included (5 and 7) were obtained to test the performance of the feedback-feedforward algorithm implemented on the nanopositioning platform. The results are plotted in Figure 5.

\section{A. Tracking performance in presence of resonance frequency variations}

Data was collected for open- and closed-loop unloaded and loaded system for $10 \mathrm{~Hz}, 40 \mathrm{~Hz}$ and $100 \mathrm{~Hz}$ scan signals including 7 odd harmonics, to verify that the feedback damping controller does make the system more robust under changes in resonance frequency,. The results are provided in Figure 6.

Change in resonance frequency due to loading is equivalent to a modeling error due to plant uncertainty. As feedforward alone cannot handle uncertainties, the loaded system when excited by the inversion-based input procured using the original model, results in poor tracking performance. On the other hand, 


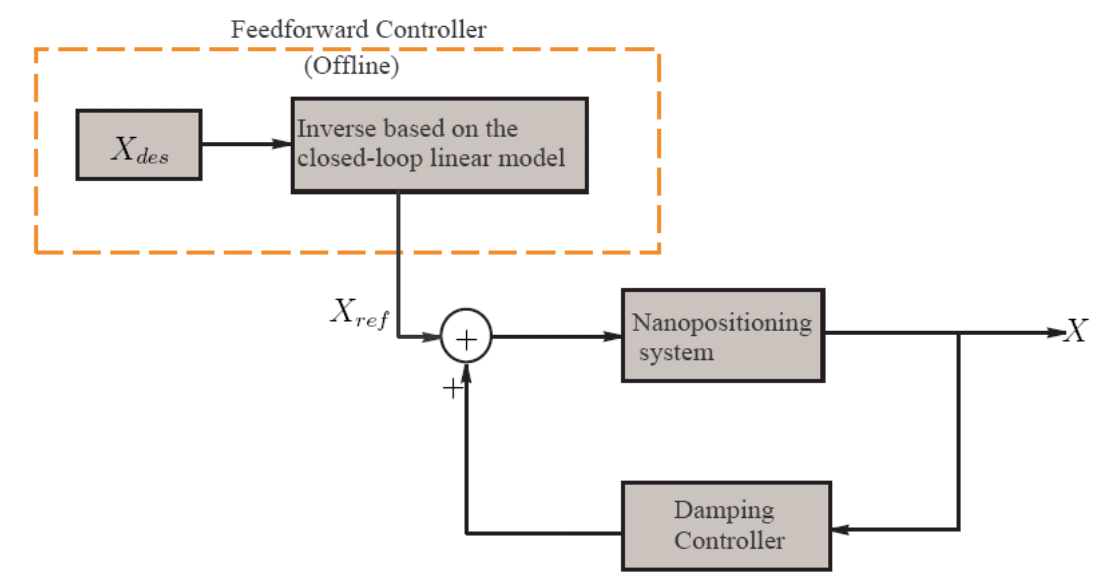

Fig. 4. Implemented control strategy. Note that positive feedback is implemented.

\begin{tabular}{|c|c|c|c|}
\hline $\begin{array}{l}\% \text { error from the perfect } \\
\text { triangle }\end{array}$ & $\begin{array}{l}\% \text { of } 10 \mathrm{~Hz}+5 \text { odd harmonics } \\
\text { within limit }\end{array}$ & $\begin{array}{l}\% \text { of } 40 \mathrm{~Hz}+5 \text { odd harmonics } \\
\text { within limit }\end{array}$ & $\begin{array}{l}\% \text { of } 100 \mathrm{~Hz}+5 \text { odd harmonics } \\
\text { within limit }\end{array}$ \\
\hline 1 & 62.4 & 59.2 & 48.7 \\
\hline 2 & 93.1 & 94.4 & 94.2 \\
\hline 5 & 100 & 100 & 100 \\
\hline 1 & 67.7 & 64.2 & 41.2 \\
\hline 2 & 96.8 & 96.8 & 95.6 \\
\hline
\end{tabular}

TABLE II

TABLE SHOWING THE PERCENTAGE OF SCAN WITHIN A PARTICULAR ERROR RANGE FOR MEASURED SCAN SIGNALS WITH THREE DIFFERENT FUNDAMENTAL FREQUENCIES.

if feedback is used to desensitize the system to resonance frequency changes, a similar scenario will yield better tracking of the desired signal. The following table presents the results of this analysis.

At relatively low frequencies $(10 \mathrm{~Hz})$, the addition of feedback only marginally improves the tracking performance of the overall system. Also, at frequencies close to the end of the bandwidth ( $7^{\text {th }}$ odd harmonic of $100 \mathrm{~Hz}$ is $1300 \mathrm{~Hz}$, the edge of achievable bandwidth), the system does not achieve perfect tracking in the presence of uncertainty. Yet, for most frequencies in between these extremes, the addition of feedback to feedforward, improves the tracking performance of the system substantially, as evident from the results at $40 \mathrm{~Hz}$ shown in Figure 6 (c) and (d).

\section{CONCLUSiOnS}

We successfully implemented the polynomial-based feedback for damping integrated with inversion-based feedforward control for tracking on a piezoelectric-stack actuated nanopositioning platform. The addition of feedback has shown to increase the positioning bandwidth of the nanopositioning platform by $1 \mathrm{kHz}$, from $310 \mathrm{~Hz}$ (using feedforward alone) to $1320 \mathrm{~Hz}$ (feedback and feedforward together). The experimental results show that a perfectly band-limited $15 \mu \mathrm{m}$ input scan signal with the fundamental frequency of $100 \mathrm{~Hz}$ (about a fourth of the resonance) and frequency components as high as 1300 $\mathrm{Hz}$ was tracked with high accuracy. It was further proved experimentally that in the presence of resonance frequency uncertainties, feedback also improves the tracking performance of the feedforward control.

\section{ACKNOWLEDGMENT}

This research was supported by the Australian Research Council's Center of Excellence for Complex Dynamic Systems and Control at the University of Newcastle, Callaghan, Australia.

\section{REFERENCES}

[1] S. Salapaka, A. Sebastin, J. P. Cleveland, and M. V. Salapaka, "High bandwidth nano-positioner: A robust control approach," Review of Scientific Instruments, vol. 73, no. 9, p. 3232 3241, 2002.

[2] M. Sitti and H. Hashimoto, "Teleoperated tough feedback from the surfaces at the nanoscale: Modeling and experiments," IEEE/ASME Trans. on Mechatronics, vol. 8, pp. 287 - 298, February 2003.

[3] A. Sebastian and S. M. Salapaka, "Design methodologies for robust nanopositioning," IEEE Trans. Contr. Syst. Tech., vol. 13, no. 6, pp. $868-876$, November 2005.

[4] M. Tomizuka, "On the design of digital tracking controllers," ASME Journal of Dynamic Systems, Measurement and Control, pp. 412 - 418, November 50th Anniversary Issue.

[5] T. Tsao, "Optimal feedforward digital tracking controller design," ASME Journal of Dynamic Systems, Measurement and Control, vol. 116, no. 4, pp. 583 - 592, December 1994.

[6] Q. Zhou and S. Devasia, "Preview-based optimal inversion for output tracking: Application to scanning tunneling microscopy," IEEE Trans. on Control System Technology, vol. 12, no. 3, pp. 375 - 386, May 2004

[7] D. Croft, S. Stilson, and S. Devasia, "Optimal tracking of piezo-based nanopositioners," Nanotechnology, vol. 10, no. 2, pp. $201-208$, June 1999.

[8] D. Croft, G. Shedd, and S. Devasia, "Creep, hysteresis, and vibration compensation for. piezoactuators: Atomic force microscopy application," Journal of Dynamic Systems, Measurement, and Control, vol. 123, no. 1, pp. 35-43, March 2001. 

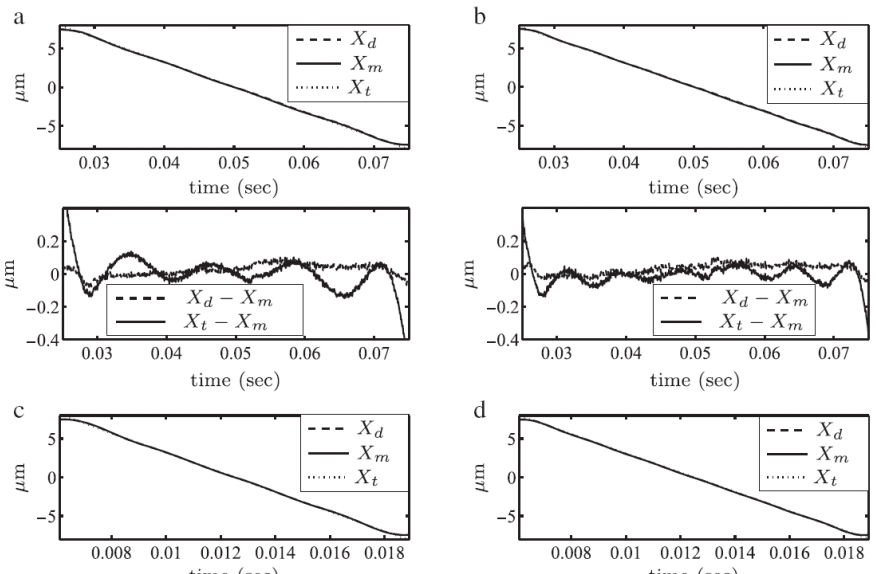
ime $(\mathrm{sec})$
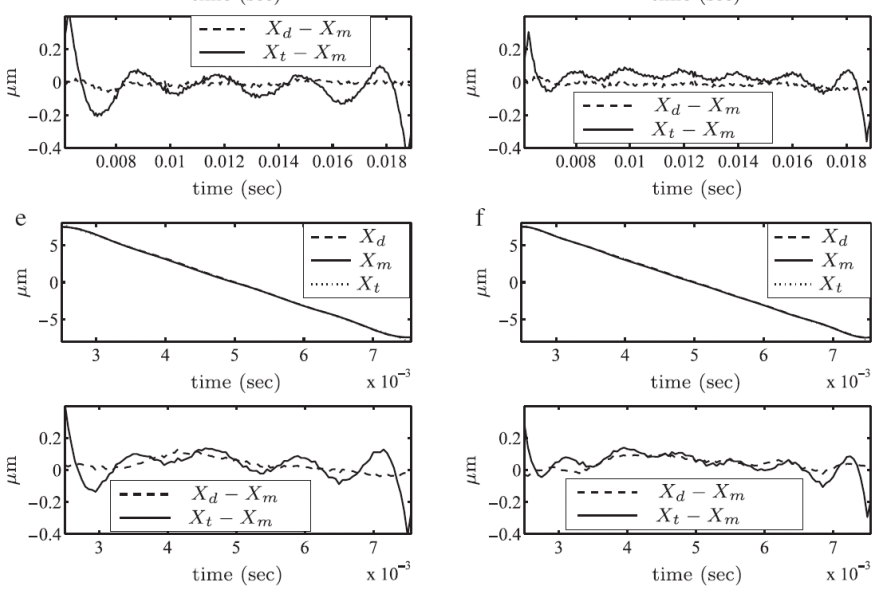

Fig. 5. Plots for the desired signal $\left(X_{d}\right)$, measured signal $\left(X_{m}\right)$ and the perfect triangle $\left(X_{t}\right)$. The respective errors $X_{t}-X_{m}$ and $X_{d}-X_{m}$ are also plotted for (a) $10 \mathrm{~Hz} 5$ harmonics, (b) $10 \mathrm{~Hz} 7$ harmonics, (c) $40 \mathrm{~Hz} 5$ harmonics, (d) $40 \mathrm{~Hz} 7$ harmonics, (e) $100 \mathrm{~Hz} 5$ harmonics and (f) $100 \mathrm{~Hz} 7$ harmonics scan signals. Note that the major portion of the error $X_{t}-X_{m}$ is due to the error in $X_{t}-X_{d}$. Sensor noise and modeling imperfections are the main causes for the error $X_{d}-X_{m}$

[9] Y. Zhao and S. Jayasuriya, "Feedforward controllers and tracking accuracy in the presence of plant uncertainties," Trans. of ASME, Journal of Dynamic Systems, Measurement and Control, vol. 117, pp. 490 - 495, December 1995.

[10] S. O. R. Moheimani, S. Devasia, and E. Eleftheriou, "Guest editorial introduction to the special issue on dynamics and control of micro- and nanoscale systems," IEEE Trans. on Control Systems Technology, vol. 15, no. 5, pp. $799-801,2007$.

[11] S. Devasia, E. Eleftheriou, and S. O. R. Moheimani, "A survey of control issues in nanopositioning," IEEE Trans. on Control Systems Technology: Special Issue on Dynamics and Control of Micro- and Nanoscale Systems., vol. 15, no. 5, pp. 802 - 823, September 2007.

[12] A. J. Fleming and S. O. R. Moheimani, "Adaptive piezoelectric shunt damping," IOP Smart Materials and Structures, vol. 12, pp. $18-28$, February 2003.

[13] D. Niederberger, A. J. Fleming, S. O. R. Moheimani, and M. Morari, "Adaptive multimode resonant piezoelectric shunt damping," Smart Materials and Structures, vol. 18, no. 2, pp. 291-315, October 2004.

[14] G. Schitter, P. Menold, H. Knapp, F. Allgower, and A. Stemmer, "High performance feedback for fast scanning atomic force microscopes,", Rev. Sci. Instrum., vol. 72, no. 8, pp. 3320 - 3327, 2001.

[15] T. Ando, N. Kodera, D. Maruyama, E. Takai, K. Saito, and A. Toda, "A high-speed atomic force microscope for studying biological macromolecules in action," Japanese Journal of Applied Physics, vol. 41, no. 7B, pp. 4851-4856, 2002.

[16] N. Kodera, H. Yamashita, and T. Ando, "Active damping of the scanner for high-speed atomic force microscopy," Rev. Sci. Instrum., vol. 76, no. 5, pp. $1-5,2005$.

[17] J. L. Fanson and T. K. Caughey, "Positive position feedback control for large space structures," AIAA Journal, vol. 28, no. 4, pp. 717 - 724, 1990.

[18] H. R. Pota, S. O. R. Moheimani, and M. Smith, "Resonant controllers
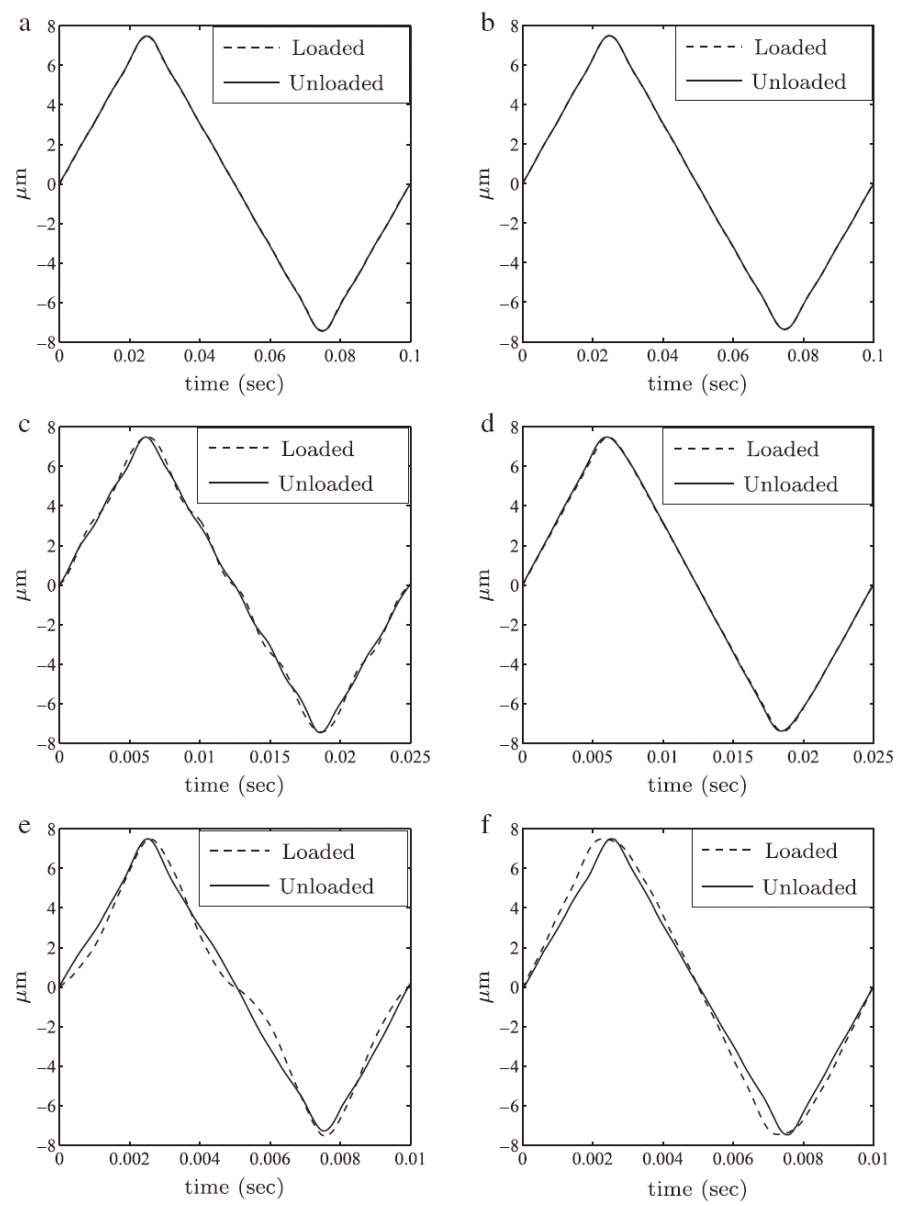

Fig. 6. (a),(c) and (d) show the open-loop (feedforward only) plots for $10 \mathrm{~Hz}$, $40 \mathrm{~Hz}$ and $100 \mathrm{~Hz}$ scan signals respectively, with first 7 odd harmonics. (b), (d) and (f) show the closed-loop (feedback and feedforward combined) tracking results for the same. Note that in closed-loop (feedback and feedforward), the tracking is visibly superior under changes in resonance frequency (loaded v.s. unloaded) than in open-loop where only feedforward is being used. As the frequency content in the scan signal increases, the scan performance of the closed-loop worsens due to an increase in the modeling errors (due to change in resonance frequency).

for smart structures," Smart Materials and Structures, vol. 11, no. 1, pp $1-8,2002$.

[19] G. C. Goodwin, S.F. Graebe and M. E. Salgado, Control System Design Prentice Hall International, Inc, 2001.

[20] S. O. R. Moheimani, B. J. G. Vautier, and B. Bhikkaji, "Experimental implementation of extended multivariable PPF control on an active structure," IEEE Trans. Contr. Syst. Tech., vol. 14, no. 3, pp. 443-455, May 2006.

[21] B. Bhikkaji, M. Ratnam, A. J. Fleming, and S. O. R. Moheimani, "Highperformance control of piezoelectric tube scanners." IEEE Trans. Control Systems Technology, vol. 5, no. 5, pp. 853 - 866, September 2007.

[22] S. S. Aphale, B. Bhikkaji, and S. O. R. Moheimani, "Minimizing scanning errors in piezoelectric stack-actuated nanopositioning platforms," IEEE Trans. on Nanotechnology, vol. 7, no. 9, pp. 79 - 90, 2008.

[23] A. Fleming and S. O. R. Moheimani, "A ground-loaded charge amplifier for reducing hysteresis in piezoelectric tube scanners," Rev. Sci. Instrum., vol. 76, no. 7, 2005 .

[24] T. McKelvey, H. Akcay, and L. Ljung, "Subspace based multivariable system identification from frequency response data," IEEE Trans. on Automatic Control, vol. 41, no. 7, pp. 960-978, July 1996.

[25] S. S. Aphale, S. Devasia, and S. O. R. Moheimani, "High-bandwidth control of a piezoelectric nanopositioning stage in the presence of plant uncertainties," Nanotechnology, vol. 19, p. 125503 (9pp), 2008.

[26] S. Devasia, "Should model-based inversion inputs be used as feedforward under plant uncertainty?" IEEE Trans. on Automatic Control, vol. 47, no. 11 , November 2002 . 